\title{
EDITORIAL
}

\section{Roles of Causality for Understanding the Behavior of System Stakeholders}

\author{
Jaemun Sim and Kyoung-Yun Kim \\ Department of Industrial and Systems Engineering, Wayne State University, Detroit, MI, USA
}

The understanding of stakeholder's behavior is essential to design a system because the system should satisfy and support stakeholders for the stakeholders to adopt the system (Jiao \& Chen, 2006). Understanding of stakeholders' behavior requires knowledge about how they work in the designed system and how they respond to the designed product. Furthermore, if we can understand why the stakeholders work or respond in such a way, we can predict the behavior of stakeholders. The causality refers to the relationship between causes and effects. The causality is essential to stakeholder behavior analysis. The causality analysis of the stakeholder behaviour contributes to the system design and analysis by providing knowledge on three perspectives (i.e., the prediction of stakeholder behavior to the new system, the motivation of the new system design, and the new system itself). Specific examples for these three perspectives are following: first, we can build a stakeholder response model. The model can be a structuralhypothetic model in social science (Biddle \& Marlin, 1987; Bagozzi \& Yi, 1988) and customer's cognition model for a product (Khalid \& Helander, 2004; Li, 2004). Second, stakeholder's dissatisfaction inferred by (or evaluated from) the model can be a motivation for a new system. Lastly, the causality of stakeholder's behavior can be implemented as an intelligent system itself. For instance, the causality can be converted into a mathematical model like operations research model (Shannon et al., 1980).

This issue gathers four papers among which the first two concentrate on stakeholder behavior prediction such as the hypothetical model between the cooperative knowledge sharing and firm's innovativeness and the customer's psychological response for a shaving product. The other two papers discuss about the healthcare visiting scheduling system motivated by practitioner privacy protection and the interpretable knowledge mining for the health care decision makers of Reusable Medical Equipment (RME) repair processes.

The first paper written by Oswaldo Jose Jimenez Torres and Dapeng Liang, entitled "Impact of Individual's Cooperative Behaviors on Innovation: Evidence from China" explores the causality between cooperative knowledge sharing behavior and firm innovativeness to design an innovative firm. This paper develops the hypothetical causal model to test how individual's knowledge sharing makes impacts on the firm's innovation capability, especially under the salience social-interaction of 'Guanxi' in China. Innovativeness of a firm is built based upon knowledge sharing dynamics among cooperative staffs. This paper constructs four hypotheses: 1) engagement in cooperative behaviors with outsiders of Guanxi; 2) trust towards outsiders to Guanxi networks; 3) years of experience of the individuals; and 4) firm's innovation capability. It confirms that the cooperative behavior with outsiders and the trust to outsiders have positive effects on the innovation of the firm while the experience year does not impact. As a result, to design an 
innovative firm, the firm needs to activate the informal interaction for easing better-connected networks and develop positive perceptions of trust toward outsiders, rather than to adopt the closed norm of Guanxi.

The second paper, entitled "Consumer-Driven Design Approach Based on Multiple Psychological Responses to Product Form" written by Hung-Yuan Chen and Kuo-Li Huang, presents the method of analysing the causality between the shape of shaver products and customer's psychological responses (CPR) to design a new shaver product. Predicted design parameters are expected to meet the aimed customer's reaction of multiple CPR (e.g., concise, individual, masculine, and vivacious). The proposed method combines two algorithms, that are Conjoint Analysis (CA) and Technique for Order of Preference by Similarity to Ideal Solution (TOPIS). CA can evaluate score in the corresponding CPR domains for specified shaver forms. TOPIS can deal with multiple-objective decision-making tasks and be used to determine the optimal design parameters of a shaver form, which achieves a specified set of four CPR values. The case study shows the proposed method's feasibility and probability of making commercial success of a product in the market.

The third paper, entitled "A Market-Based Scheduling Mechanism Design for Cost Reduction in Home Health Care" written by Jie Gao, Zhijie Xie and Chun Wang, is about how to design new scheduling systems of home health care visit, motivated by the privacy protection of practitioners, while reducing the health care agency's overall cost and increasing practitioners' payment for health care visiting. This paper implements the iterative auction mechanism-based scheduling by the causality of the overall reaction between two stakeholders (i.e., service provider and service practitioner). In the iterative auction, practitioners update their bidding prices for their feasible services, and the agency calculates the optimal schedule allocation with two constraints (i.e., minimizing overall payment and satisfying customer's requirements). To validate the proposed method, the iterative auction model is compared with the optimized solution as centralized scheduling, in which the practitioner needs to reveal their entire private information.

The last paper, entitled "Semantic and Association Rule Mining-based Knowledge Extension for Reusable Medical Equipment Random Forest Rules" written by Jong Youl Lee and Kyoung-Yun Kim, presents a knowledge interpretation method to convert complex rules that are generated by machine running algorithms to human-readable rules. The rule aims to help to decide the repair schedule of Reusable Medical Equipment (RME). RME enables healthcare providers to reduce cost by reusing and reprocessing the equipment device; however, it requires periodical repairs. Random forest is a useful algorithm due to its high prediction accuracy of scheduling repair tasks; however, it generates tremendous rules to capture all possible repair tasks from RME repair history data. The healthcare provider needs to understand the causality to mitigate the uncertainty of the prediction results, because all prediction results from the algorithm are not necessarily perfect. Currently, it is impossible for a human-decision maker to understand the causality behind tremendous rule sets. The article shows that a post-mining approach combined with association rule mining reduces the number of rules by mining the compact subset of rules with important priority. Moreover, it shows the method of human-readable knowledge extension by combining RME domain ontology.

The causality is essential for building a decision model and designing an intelligent system. In the age of data, advanced data mining technology (e.g., big data) has been highlighted and can extract potentially causality from data. However, data mining technology is still limited because of its limited data measurement capability. It tends to generate inaccurate causality and ends up resulting in an untrusted system. Also, when the causality comes from a black box model, this causes a limited model-interpretation capability. For this reason, a system user often experiences a trust uncertainty of the results. The black box model cannot provide reasonable information about the rationality of the extracted causality. The causality models using hypothetical model, CPR, auction mechanism, etc. can complement the causality models constructed by data mining algorithms. However, significant research works have not been conducted to take advantage of this capability. More research works are therefore required to understand how the 
uncertainty of causality models can be reduced and how the models can support the reasoning rationality for the system design and increase the system trust.

\section{References}

Jiao, J. and Chen, C.H. (2006). Customer requirement management in product development: a review of research issues. Concurrent Engineering, 14(3), pp.173-185. doi: 10.1177/1063293X06068357

Biddle, B.J. and Marlin, M.M. (1987). Causality, confirmation, credulity, and structural equation modeling. Child development, pp.4-17. doi: 10.2307/1130287

Bagozzi, R.P. and Yi, Y. (1988). On the evaluation of structural equation models. Journal of the academy of marketing science, 16(1), pp.74-94. doi: 10.1007/BF02723327

Shannon, R.E., Long, S.S. and Buckles, B.P. (1980). Operation research methodologies in industrial engineering: A survey. AIIE Transactions, 12(4), pp.364-367. doi: 10.1080/05695558008974528

Khalid, H.M. and Helander, M.G. (2004). A framework for affective customer needs in product design. Theoretical Issues in Ergonomics Science, 5(1), pp.27-42. doi: 10.1080/1463922031000086744

Lin, I.Y. (2004). Evaluating a servicescape: the effect of cognition and emotion. International Journal of Hospitality Management, 23(2), pp.163-178. doi: 10.1016/j.ijhm.2003.01.001

\section{Author Biography}

Dr. Jaemun Sim is a post-doc fellow at Wayne State University since 2016. Dr. Sim obtained his Ph.D in Kyung Hee University, South Korea. He also worked as a post-doc at Sungkyunkwan University, South Korea. He has published multiple articles in leading decision science journals, such as Expert Systems with Applications. Dr. Sim's main research interests include human-computer interaction, creativity science, and artificial intelligence applications in business, traffic systems, energy consumption, emotional space service, health care service, and manufacturing. Recently, Dr. Sim is involved in Computational Intelligence and Design Informatics (CInDI) Laboratory and the Michigan Translational Research and Commercialization (MTRAC) project. He works on the development of Virtually Guided RSW Weldability Prediction (VRWP) platform, specifically joinability prediction for the manufacturing industry.

Dr. Kyoung-Yun Kim is a professor in the Department of Industrial and Systems Engineering at Wayne State University, where he directs the Computational Intelligence and Design Informatics (CInDI) Laboratory. Dr. Kim's research focuses on Design Science; Design Informatics; Semantic Assembly Design; Welding and Joining; and Smart Manufacturing. Currently, Dr. Kim is Director of Smart Manufacturing Demonstration Center (SMDC) and a Site Director for the NSF Industry and University Cooperative Research Center (I/UCRC) for e-Design. Dr. Kim is a Fellow of the Society of Design and Process Science. Dr. Kim received top cited article award (2005-2010) from Journal CAD and 2003 IIE Transactions Best Paper Award. Dr. Kim was an invited professor at Université de Technologie de BelfortMontbéliard (UTBM), France, 2017 and was a visiting professor at Kyung Hee University, South Korea, 2014. 